TITLE:

\title{
Electron-phonon coupling in negatively charged acene- and phenanthrene-edge-type hydrocarbon crystals
}

$\operatorname{AUTHOR}(\mathrm{S}):$

Kato, T; Yoshizawa, K; Hirao, K

\section{CITATION:}

Kato, T ... [et al]. Electron-phonon coupling in negatively charged acene- and phenanthrene-edge-type hydrocarbon crystals. JOURNAL OF CHEMICAL PHYSICS 2002, 116(8): 3420-3429

ISSUE DATE:

2002-02-22

URL:

http://hdl.handle.net/2433/39736

\section{RIGHT:}

Copyright 2002 American Institute of Physics. This article may be downloaded for personal use only. Any other use requires prior permission of the author and the American Institute of Physics. 


\title{
Electron-phonon coupling in negatively charged acene- and phenanthrene-edge-type hydrocarbon crystals
}

\author{
Takashi Kato ${ }^{\mathrm{a}}$ \\ Institute for Fundamental Chemistry, 34-4 Takano-Nishihiraki-cho, Sakyo-ku, Kyoto 606-8103, Japan
}

Kazunari Yoshizawa

Institute for Fundamental Research of Organic Chemistry, Kyushu University, Higashi-ku, Fukuoka 812-8581, Japan

Kazuyuki Hirao

Department of Material Chemistry, Graduate School of Engineering, Kyoto University, Sakyo-ku, Kyoto 606-8501, Japan

(Received 15 October 2001; accepted 29 November 2001)

\begin{abstract}
Vibronic interaction and its role in the occurrence of possible superconductivity in the monoanions of phenanthrene-edge-type aromatic hydrocarbons are studied. The vibrational frequencies and the vibronic coupling constants are computed and analyzed and the electron-phonon coupling constants are estimated. The results for phenanthrene-edge-type hydrocarbons are compared with those for acene-edge-type hydrocarbons. The lowest frequency mode and the $\mathrm{C}-\mathrm{C}$ stretching modes of $1400-1600 \mathrm{~cm}^{-1}$ afford large electron-phonon coupling constants in the monoanions of acene- and phenanthrene-edge-type hydrocarbons. The total electron-phonon coupling constants decrease with an increase in the number of carbon atoms in both acene- and phenanthrene-edge-type hydrocarbons, but those for the monoanions of phenanthrene-edge-type hydrocarbons are larger than those for the monoanions of acene-edge-type hydrocarbons. Possible superconducting transition temperatures $T_{\mathrm{c}} \mathrm{s}$ for the monoanions are estimated. The monoanions of phenanthrene-edge-type hydrocarbons would have higher $T_{\mathrm{c}} \mathrm{s}$ than the monoanions of acene-edgetype hydrocarbons if phenanthrene-edge-type hydrocarbons exhibit superconductivity. These results suggest that molecular edge structures as well as molecular sizes have relevance to the strength of electron-phonon coupling and $T_{\mathrm{c}} \mathrm{s}$. The fragment molecular-orbital method (FMO) method successfully characterizes the distinct electronic structures of the two small polynuclear aromatic hydrocarbons (PAHs) with different type of edges such as anthracene and phenanthrene. (C) 2002 American Institute of Physics. [DOI: 10.1063/1.1445102]
\end{abstract}

\section{INTRODUCTION}

Superconductivity or the presence of zero resistance towards the passage of an electrical current is a very interesting property of many solids. Early ideas about conventional superconductivity culminated in a theory put forward by Bardeen, Cooper, and Schrieffer (BCS) (Refs. 1, 2) in 1957. According to the BCS theory, the interaction of conduction electrons with phonons ${ }^{3-5}$ leads to an attraction between the normally repulsive electrons so that they can pair up and flow without resistance. However, we cannot at present still define the connection between structure and superconductivity because the microscopic mechanisms for the phenomenon remains unsolved for many systems such as hightemperature cuprates.

Batlogg et al. ${ }^{6}$ reported complete switching between the insulating and superconducting states in the fullerene fieldeffect transistor (FET). A series of acenes such as anthracene $\left(\mathrm{C}_{14} \mathrm{H}_{10}\right)$, tetracene $\left(\mathrm{C}_{18} \mathrm{H}_{12}\right)$, and pentacene $\left(\mathrm{C}_{22} \mathrm{H}_{14}\right)$ were recently found to exhibit superconductivity at low

a) Author to whom correspondence should be addressed Present address: Max-Planck-Institut für Festkörperforschung, Heisenbergstr. 1, D-70569 Stuttgart, Germany. Electronic mail: kato@ifc.or.jp, t.kato@fkf.mpg.de temperatures. ${ }^{7}$ The superconducting transition temperatures $T_{\mathrm{c}} \mathrm{s}$ increase from 2 to $4 \mathrm{~K}$ with a decrease in molecular size from pentacene to anthracene. Such a new class of superconductors is insulating organic crystals that are made metallic through charge injection by using FET geometry. The electron density should correspond to about one electron per molecule, assuming that only the topmost molecular layer takes part in conduction. ${ }^{8}$ Several researchers proposed that the charge transport in the crystals of naphthalene $\left(\mathrm{C}_{10} \mathrm{H}_{8}\right)$, anthracene, tetracene, and pentacene is dominated by electron-phonon interactions. ${ }^{9-12}$ Interestingly, possible superconductivity of polyacenes has been proposed from a theoretical viewpoint. ${ }^{13,14}$ Batlogg et al. recently observed gateinduced superconductivity in hole- and electron-doped $\mathrm{C}_{60}$ single-crystal FET structures. ${ }^{15}$ Pure intramolecular Ramanactive modes are suggested to be important in a BCS-type strong coupling scenario in superconductivity in fullerenes. ${ }^{16}$

It has been clarified from theoretical studies at various levels that the electronic properties of highly condensed polynuclear aromatic hydrocarbons (PAHs) significantly depend on their molecular sizes and edge structures. ${ }^{17-22}$ There are several prototypes for the edge structures of PAHs: one of the most important edge structures is "phenanthrene- 


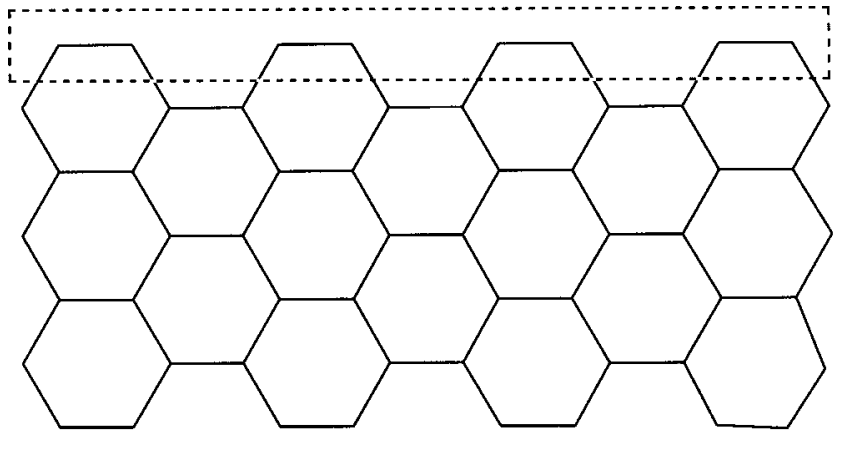

phenanthrene-edge-type

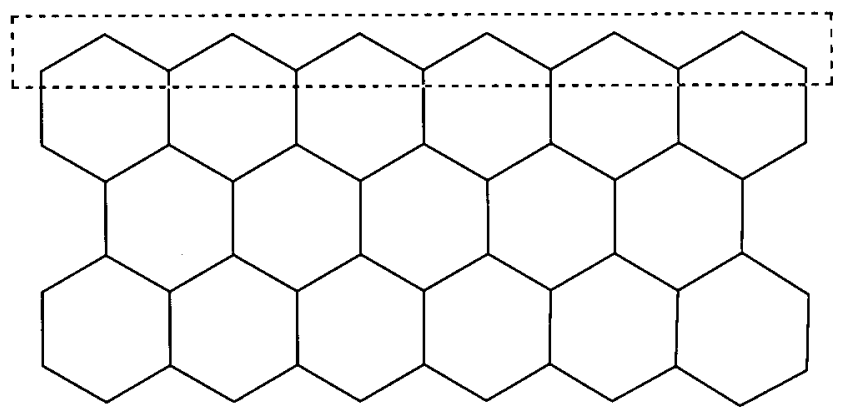

acene-edge-type

Scheme 1

edge-type" (or armchair-edge-type) and another is "aceneedge-type" (or zig-zag-edge-type), as shown in Scheme 1. The characteristics of the frontier orbitals, the orbitals in the vicinity of the Fermi level, significantly depend on the edge structures of PAHs. ${ }^{19,21}$ In the acene-edge structure, the frontier orbitals are localized at the edges, whereas in the phenanthrene-edge structure the frontier orbitals are distributed over the carbon structure. The characterization of the electronic structures of anthracene and phenanthrene is a classical problem in quantum chemistry. Although both anthracene and phenanthrene have the same molecular formula of $\mathrm{C}_{14} \mathrm{H}_{10}$, their electronic properties are quite different, due to their structural differences. Fukui ${ }^{23}$ and Hosoya ${ }^{24,25}$ explained the reason why phenanthrene is energetically more stable than anthracene. It is intriguing to study how the structural differences cause the different properties of the vibronic interactions and electron-phonon interactions between acene- and phenanthrene-edge-type hydrocarbons.

In view of the interesting molecular superconductivity in acenes and fullerene, we can reasonably expect that phenanthrene-edge-type hydrocarbon crystals such as phenanthrene $\left(\mathrm{C}_{14} \mathrm{H}_{18}\right)$, chrysene $\left(\mathrm{C}_{18} \mathrm{H}_{12}\right)$, and benzo[a]chrysene $\left(\mathrm{C}_{22} \mathrm{H}_{14}\right)$ might also exhibit superconductivity caused by vibronic interactions between molecular vibrations and frontier orbitals. In this study, we first calculate the orbital vibronic coupling constants which are characterized by intramolecular properties in phenanthrene-edge-type hydrocarbons. We next calculate the electron-phonon coupling constants for the monoanions of phenanthrene-edge-type hydrocarbons in the framework of a one-electron approximation. We compare the electron-phonon coupling in the monoanions of phenanthrene-edge-type hydrocarbons with that in the monoanions of acene-edge-type hydrocarbons studied in a previous research. ${ }^{26}$ Next, we predict possible $T_{\mathrm{c}} \mathrm{s}$ for the monoanions of phenanthrene-edge-type hydrocarbon crystals. We derive structure-property relationships in the predicted $T_{\mathrm{c}} \mathrm{s}$.

\section{THEORETICAL BACKGROUND}

We describe the theoretical background for the vibronic coupling in phenanthrene-edge-type hydrocarbons. That for acene-edge-type hydrocarbons was described in the previous research. ${ }^{26}$ We will use small letters for "one-electron orbital symmetries" and capital letters for symmetries of both "electronic" and "vibrational" states, as usual. The vibronic matrix element, $E_{x y}(r, Q),{ }^{3-5}$ is given by

$$
\begin{aligned}
E_{x y}(r, Q) & =\epsilon_{x y}(r, Q)-\epsilon_{x y}(r, 0) \\
& =\sum_{\alpha}\left(\frac{\partial \epsilon_{x y}}{\partial Q_{\alpha}}\right)_{0} Q_{\alpha}+\frac{1}{2} \sum_{\alpha, \beta}\left(\frac{\partial^{2} \epsilon_{x y}}{\partial Q_{\alpha} \partial Q_{\beta}}\right)_{0} Q_{\alpha} Q_{\beta},
\end{aligned}
$$

where $\epsilon_{x y}(r, Q)$ is defined as

$$
\epsilon_{x y}(r, Q)=\left\langle\phi_{x}|h(r, Q)| \phi_{y}\right\rangle .
$$

Here, $h(r, Q)$ is the Hamiltonian of one-electron orbital energy, and $\phi_{x}$ and $\phi_{y}$ are one-electron wave functions. $r$ and $Q$ signify the whole set of coordinates of the electrons and nuclei, respectively. What we see in the first term on the right-hand side of Eq. (1) is the linear orbital vibronic coupling constant.

\section{A. Vibronic interactions between the nondegenerate lowest unoccupied molecular orbitals (LUMO) and the totally symmetric vibrational modes in phenanthrene-edge-type hydrocarbons}

Let us look into orbital vibronic coupling in phenanthrene-edge-type hydrocarbons. The symmetry labels of the LUMO of phenanthrene $\left(C_{2 v}\right)$, chrysene $\left(C_{2 h}\right)$, and benzo[a]chrysene $\left(C_{2 \nu}\right)$ are $a_{2}, b_{g}$, and $a_{2}$, respectively. Direct product of the LUMO symmetry can be reduced as

$$
\begin{aligned}
& a_{2} \times a_{2}=A_{1} \quad \text { for phenanthrene and benzo[a]cyrysene, } \\
& b_{g} \times b_{g}=A_{g} \quad \text { for chrysene. }
\end{aligned}
$$

Therefore, the totally symmetric $A_{1}$ and $A_{g}$ modes couple to the LUMO. The symmetry labels of $A_{1}$ and $A_{g}$ are abbreviated as " $A$ " in the following discussion. The numbers of such totally symmetric modes are 23,29 , and 35 for phenanthrene, chrysene, and benzo[a]chrysene, respectively. The dimensionless linear orbital vibronic coupling constants of the LUMO for its $m$ th mode is defined by Eq. (5),

$$
g_{\text {LUMO }}\left(\omega_{m}\right)=\frac{1}{\hbar \omega_{m}}\left\langle\text { LUMO }\left\|\left(\frac{\partial h}{\partial q_{A_{m}}}\right)_{0}\right\| \text { LUMO }\right\rangle \text {. }
$$

In this equation, $q_{A_{m}}$ is the dimensionless normal coordinate $^{27}$ of the $m$ th mode defined by the normal coordinate $Q_{A_{m}}$ as

$$
q_{A_{m}}=\sqrt{\omega_{m} / \hbar} Q_{A_{m}} .
$$




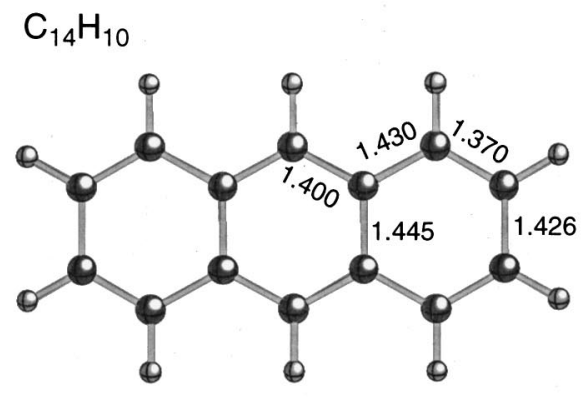

anthracene

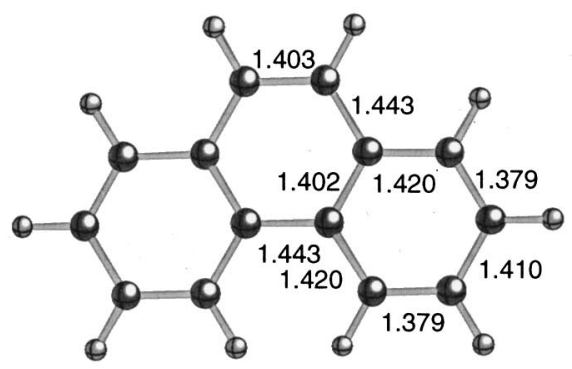

phenanthrene

$\mathrm{C}_{18} \mathrm{H}_{12}$

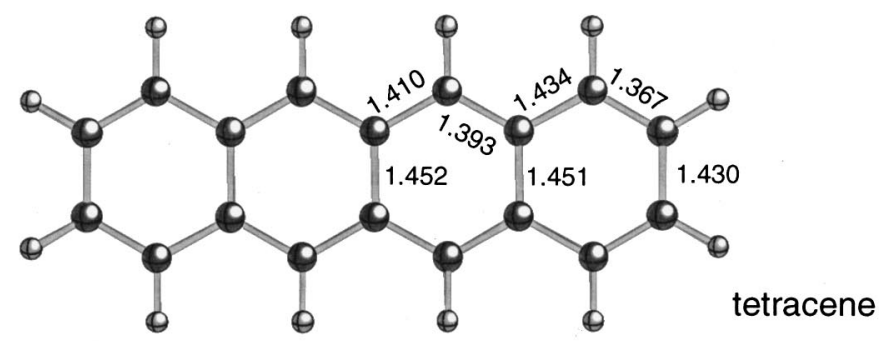

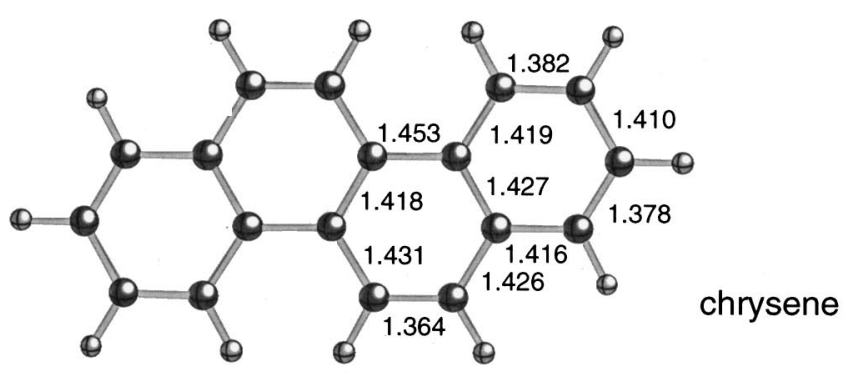

FIG. 1. Optimized structures of aceneand phenanthrene-edge-type hydrocarbons.

\section{$\mathrm{C}_{22} \mathrm{H}_{14}$}

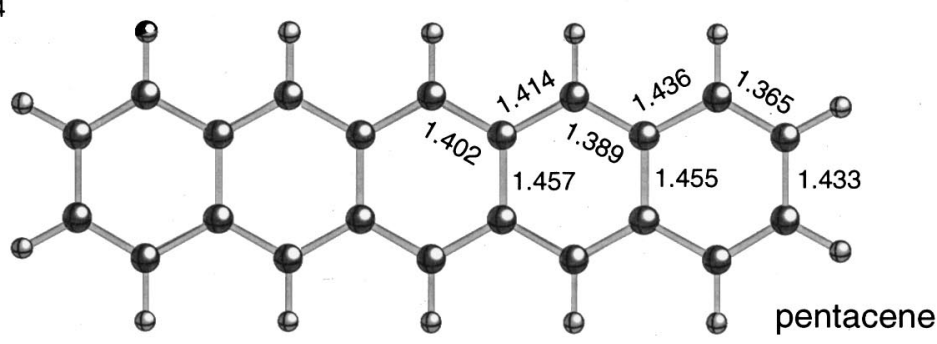

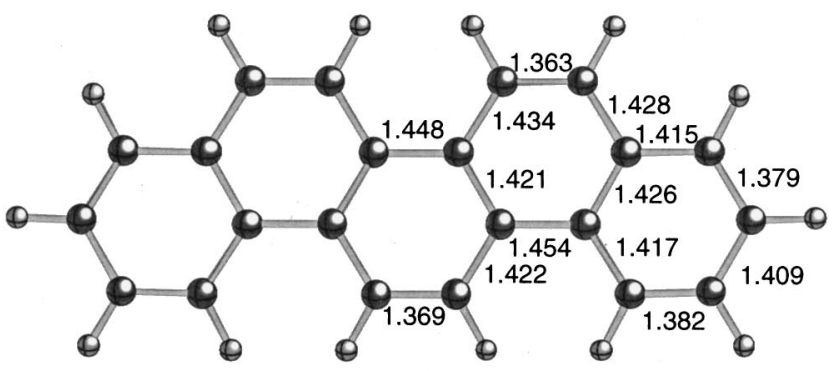

benzo[a]chrysene

\section{B. Electron-phonon coupling constants in the monoanions of phenanthrene-edge-type hydrocarbons}

We set up some assumptions to apply the calculated vibronic coupling constants to the solid-state properties of phenanthrene-edge-type hydrocarbon crystals. We first use a molecular orbital approximation for the band structure of the anion crystals in order to estimate electron-phonon coupling constants. We next assume that the conduction band of the anion crystals mainly consists of LUMO of free phenanthrene-edge-type hydrocarbons. We can derive the dimensionless electron-phonon coupling constant $\lambda$ (Refs. 16, 


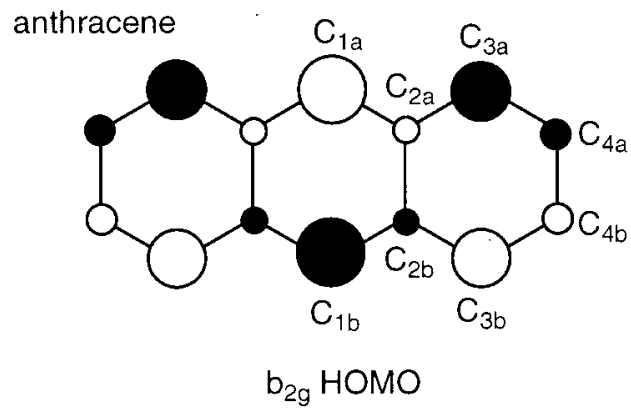

phenanthrene

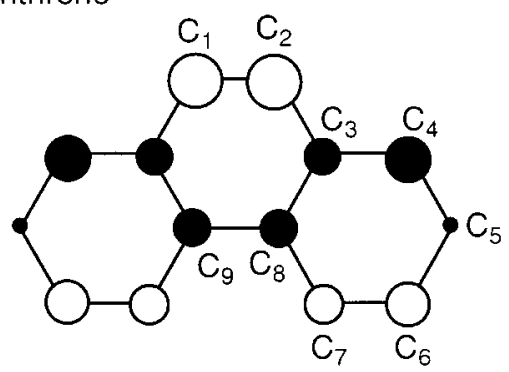

$\mathrm{b}_{1} \mathrm{HOMO}$

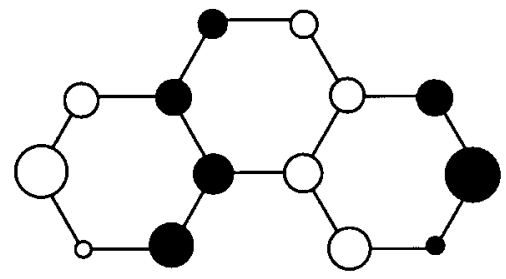

$\mathrm{a}_{2} \mathrm{HOMO}-1$

FIG. 2. Occupied frontier orbitals of anthracene and phenanthrene.

26) in the monoanions of phenanthrene-edge-type hydrocarbon crystals,

$$
\lambda=\sum_{m} \lambda_{m}, \lambda_{m}=n(0) l_{\mathrm{LUMO}}\left(\omega_{m}\right),
$$

where $n(0)$ is the density of states at the Fermi level per eV, per spin, and per molecule, and $l_{\mathrm{LUMO}}\left(\omega_{m}\right)$ is the electronphonon coupling constant defined as

$$
l_{\text {LUMO }}\left(\omega_{m}\right)=g_{\text {LUMO }}^{2}\left(\omega_{m}\right) \hbar \omega_{m} .
$$

\section{RESULTS AND DISCUSSION}

\section{A. Optimized structure}

The structures of neutral phenanthrene, chrysene, and benzo[a]chrysene were optimized under $C_{2 \nu}, C_{2 h}$, and $C_{2 \nu}$ symmetries, respectively, using the hybrid Hartree-Fock (HF)/density-functional-theory (DFT) method of Becke ${ }^{28}$ and Lee, Yang, and $\operatorname{Parr}^{29}$ (B3LYP), and the 6-31G* basis set. $^{30}$ The GAUSSIAN 98 program package ${ }^{31}$ was used for our theoretical analyses. This level of theory is, in our experience, sufficient for reasonable descriptions of the geometric and vibrational features of hydrocarbons. Optimized structures of acene- and phenanthrene-edge-type hydrocarbons are shown in Fig. 1. Each structure was confirmed from vi-

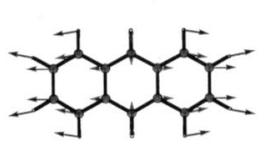

$399 \mathrm{~cm}^{-1}$

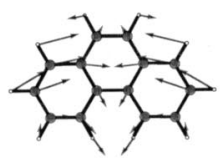

$413 \mathrm{~cm}^{-1}$

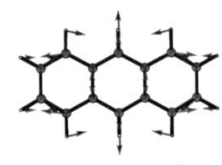

$1445 \mathrm{~cm}^{-1}$

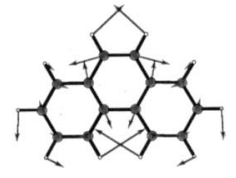

$1434 \mathrm{~cm}^{-1}$

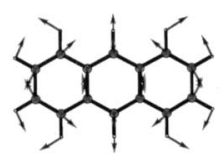

$1610 \mathrm{~cm}^{-1} \quad A_{g}$

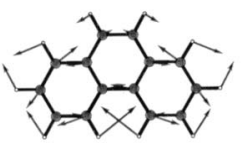

$1670 \mathrm{~cm}^{-1} \quad A_{1}$

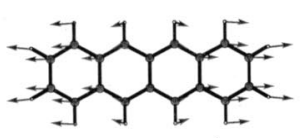

$318 \mathrm{~cm}^{-1}$

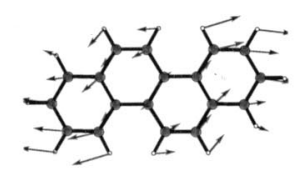

$296 \mathrm{~cm}^{-1}$

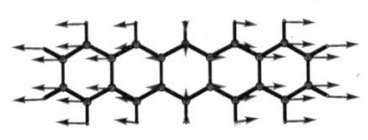

$264 \mathrm{~cm}^{-1}$

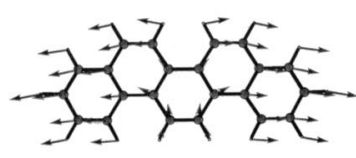

$262 \mathrm{~cm}^{-1}$
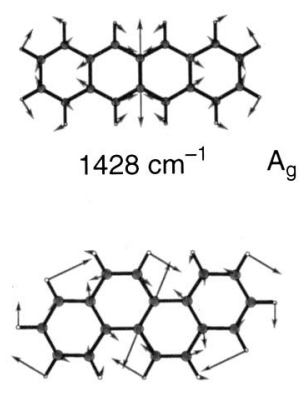

$1409 \mathrm{~cm}^{-1} \quad A_{g}$
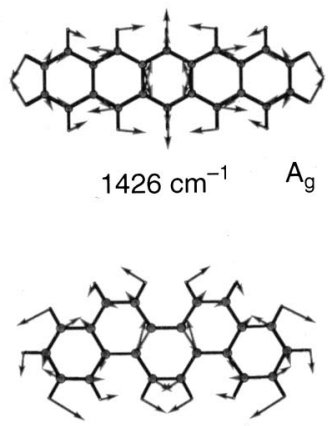

$1396 \mathrm{~cm}^{-1} \quad A_{1}$
FIG. 3. Selected vibrational modes of acene- and phenanthrene-edge-type hydrocarbons.

brational analysis to be a minimum on each potential energy surface. Occupied frontier orbitals of anthracene and phenanthrene are shown in Fig. 2. According to our calculations, the energy difference between the highest occupied molecular orbital (HOMO) and the LUMO of phenanthrene $(4.667 \mathrm{eV})$ is larger than that of anthracene $(3.593 \mathrm{eV})$, as expected. Optimized structures of phenanthrene, chrysene, and benzo[a]chrysene are more stable in energy than those of anthracene, tetracene, and pentacene by 2.5, 10.2, and 16.5 $\mathrm{kcal} / \mathrm{mol}$, respectively. The monoanions of phenanthreneedge-type hydrocarbons, however, are expected to be energetically less stable than the monoanions of acene-edge-type hydrocarbons because the LUMOs of phenanthrene-edgetype hydrocarbons lie higher in energy than those of aceneedge-type hydrocarbons. We can see from Fig. 1 that in the $D_{2 h}$ structure of anthracene, there is a distinct variation in the $\mathrm{C}-\mathrm{C}$ distances. This result is reasonable in view of the orbital patterns of the HOMOs shown in Fig. 2. This illustra- 

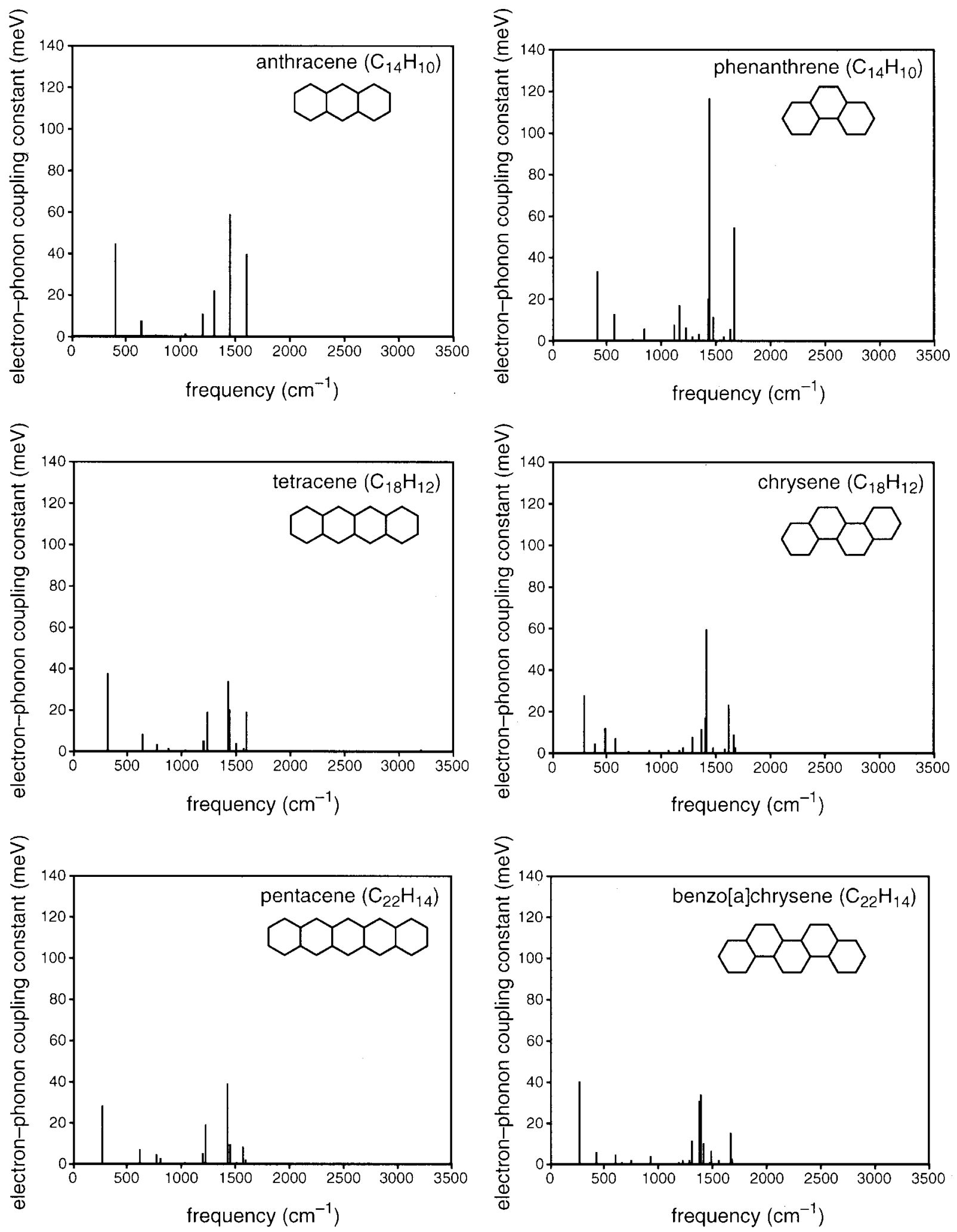

FIG. 4. Electron-phonon coupling constants $\left[l_{\mathrm{LUMO}}\left(\omega_{m}\right)\right]$ for the monoanions of acene- and phenanthrene-edge-type hydrocarbons.

tion demonstrates that the atomic orbitals between two neighboring $\mathrm{C}_{1 \mathrm{a}}$ and $\mathrm{C}_{2 \mathrm{a}}$, and $\mathrm{C}_{3 \mathrm{a}}$ and $\mathrm{C}_{4 \mathrm{a}}$ are combined in phase and thus form strong $\pi$-bonding in the HOMO of anthracene. On the other hand, the HOMO contributes to antibonding interactions between $\mathrm{C}_{2 \mathrm{a}}$ and $\mathrm{C}_{3 \mathrm{a}}, \mathrm{C}_{2 \mathrm{a}}$ and $\mathrm{C}_{2 \mathrm{~b}}$, and $\mathrm{C}_{4 \mathrm{a}}$ and $\mathrm{C}_{4 \mathrm{~b}}$. This can explain why the $\mathrm{C}_{1 \mathrm{a}}-\mathrm{C}_{2 \mathrm{a}}(1.400 \AA)$ and $\mathrm{C}_{3 \mathrm{a}}-\mathrm{C}_{4 \mathrm{a}}$ bonds $(1.370 \AA)$ are shorter than the $\mathrm{C}_{2 \mathrm{a}}-\mathrm{C}_{3 \mathrm{a}}$ $(1.430 \AA), \mathrm{C}_{2 \mathrm{a}}-\mathrm{C}_{2 \mathrm{~b}}(1.445 \AA)$, and $\mathrm{C}_{4 \mathrm{a}}-\mathrm{C}_{4 \mathrm{~b}}$ bonds $(1.426$ $\AA$ ). Similar discussions can be made in phenanthrene. The $\mathrm{C}-\mathrm{C}$ bond distances between two neighboring $\mathrm{C}$ atoms whose atomic orbitals are combined in phase in the HOMO are short $\left[\mathrm{C}_{1}-\mathrm{C}_{2}(1.403 \AA), \mathrm{C}_{3}-\mathrm{C}_{8}(1.402 \AA), \mathrm{C}_{4}-\mathrm{C}_{5}\right.$ 

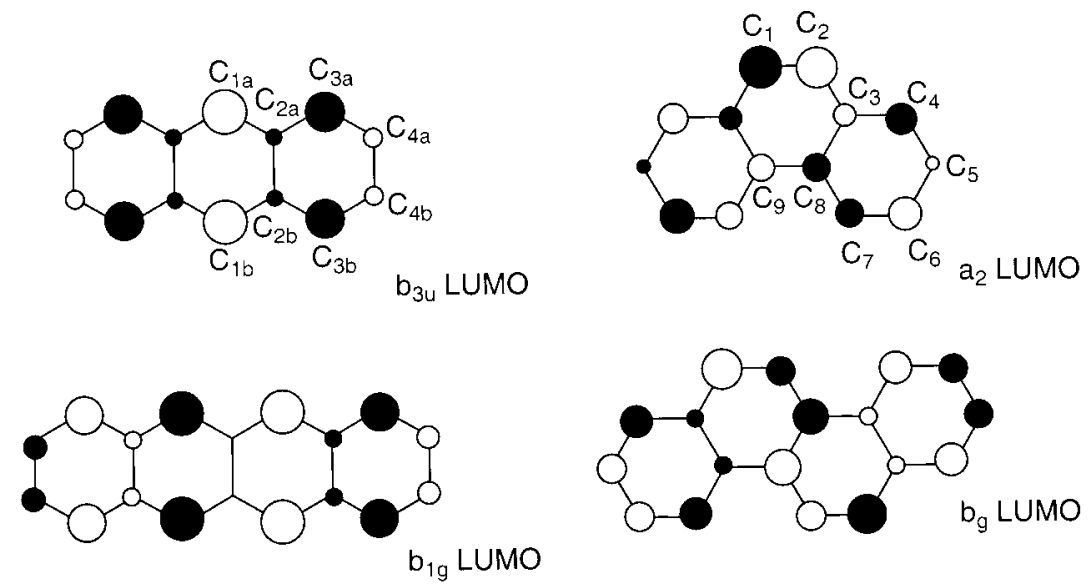

FIG. 5. The LUMOs of acene- and phenanthrene-edge-type hydrocarbons.
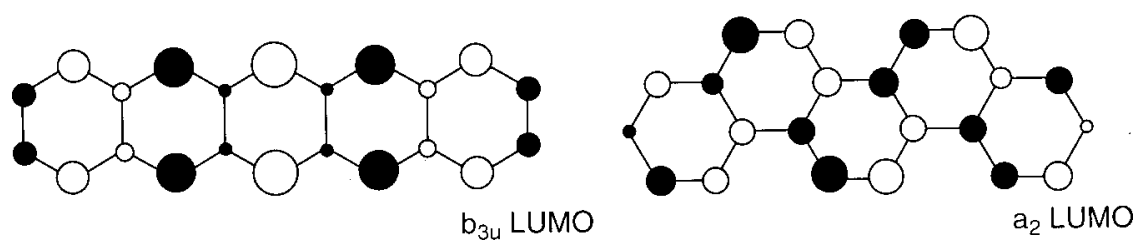

$(1.379 \AA)$, and $\left.\mathrm{C}_{6}-\mathrm{C}_{7}(1.379 \AA)\right]$, whereas those between two neighboring $\mathrm{C}$ atoms whose atomic orbitals are combined out of phase in the HOMO are long $\left[\mathrm{C}_{2}-\mathrm{C}_{3}(1.443 \AA)\right.$, $\mathrm{C}_{5}-\mathrm{C}_{6}(1.410 \AA)$, and $\left.\mathrm{C}_{7}-\mathrm{C}_{8}(1.420 \AA)\right]$. It should be noted that the $\mathrm{C}_{3}-\mathrm{C}_{4}(1.420 \AA)$ and $\mathrm{C}_{8}-\mathrm{C}_{9}(1.443 \AA)$ bonds are significantly long even though the HOMO contributes to $\pi$ bonding interactions between these two neighboring carbon atoms. This is because the HOMO- 1 , which lies only $0.32 \mathrm{eV}$ below the HOMO, also plays an essential role in the determination of the structure of phenanthrene. In fact, the HOMO-1 contributes strong $\pi$ antibonding interactions between $\mathrm{C}_{3}$ and $\mathrm{C}_{4}$, and $\mathrm{C}_{8}$ and $\mathrm{C}_{9}$.

\section{B. Orbital vibronic interactions and electron-phonon coupling in the monoanions of acene- and phenanthrene-edge-type hydrocarbons}

We carried out vibrational analyses of these acene- and phenanthrene-edge-type hydrocarbons at the B3LYP/6-31G* level of theory. Selected vibrational modes of these molecules are shown in Fig. 3. We next calculated first-order derivatives at this equilibrium structure on each orbital energy surface by distorting the molecule along the totally symmetric modes of these molecules in order to obtain orbital vibronic coupling constants $g_{\text {LUMO }}\left(\omega_{m}\right)$. We can estimate the electron-phonon coupling constants $l_{\text {LUMO }}\left(\omega_{m}\right)$ from the dimensionless diagonal linear orbital vibronic coupling constants by using Eq. (8). The calculated electronphonon coupling constants in the monoanions of acene- and phenanthrene-edge-type hydrocarbons are shown in Fig. 4. The LUMOs of these molecules are shown in Fig. 5.

Let us take a look at the electron-phonon coupling of $A_{1}$ vibrational modes to $a_{2}$ LUMO in phenanthrene. We see from Fig. 4 that the $\mathrm{C}-\mathrm{C}$ stretching $A_{1}$ mode of $1434 \mathrm{~cm}^{-1}$ strongly couples to the $a_{2}$ LUMO in phenanthrene. When phenanthrene is distorted along this $A_{1}$ mode shown in Fig. 3 , the antibonding interactions between $C_{1}$ and $C_{2}$, and $C_{3}$ and $\mathrm{C}_{8}$ in the $a_{2}$ LUMO become weak, and the bonding interaction between $\mathrm{C}_{2}$ and $\mathrm{C}_{3}$ becomes strong. Therefore, the $a_{2}$ LUMO is significantly stabilized in energy by such a distortion. On the other hand, when phenanthrene is distorted toward the opposite direction along the arrow of this mode, the $a_{2}$ LUMO is significantly destabilized in energy. This orbital-vibration relationship explains why the $\mathrm{C}-\mathrm{C}$ stretching $A_{1}$ mode of $1434 \mathrm{~cm}^{-1}$ strongly couples to the $a_{2}$ LUMO in phenanthrene. In a similar way, the $\mathrm{C}-\mathrm{C}$ stretching $A_{1}$ mode of $1670 \mathrm{~cm}^{-1}$ strongly couples to the $a_{2}$ LUMO in phenanthrene. Let us next look at the electron-phonon coupling of the $A_{g}$ vibrational modes to the $b_{3 u}$ LUMO in anthracene. Figure 4 demonstrates that the $\mathrm{C}-\mathrm{C}$ stretching $A_{g}$ modes of 1445 and $1610 \mathrm{~cm}^{-1}$ and the lowest frequency $A_{g}$ mode of $399 \mathrm{~cm}^{-1}$ strongly couple to the $b_{3 u}$ LUMO. It is worth noting that the electron-phonon coupling constants for

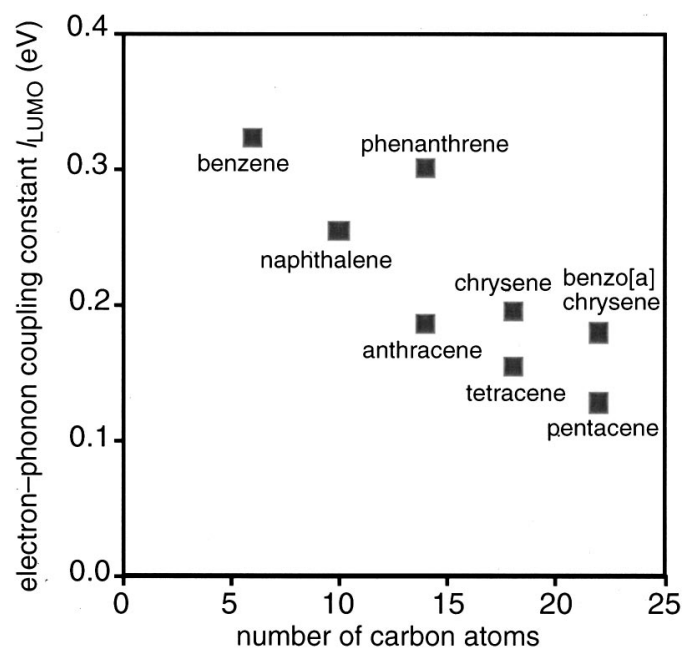

FIG. 6. Electron-phonon coupling constants $l_{\text {LUMO }}$ as a function of the number of carbon atoms. 
TABLE I. Superconducting transition temperatures $T_{\mathrm{c}} \mathrm{s}$ (in $\mathrm{K}$ ) for anthracene, tetracene, and pentacene as a function of $n(0)$ and $\mu^{*}$.

\begin{tabular}{cccccc}
\hline \hline$n(0)$ & 2.0 & 2.5 & 3.0 & 3.5 & 4.0 \\
\hline $\begin{array}{c}\text { anthracene } \\
\mu^{*}\end{array}$ & 0.099 & 0.154 & 0.204 & 0.250 & 0.292 \\
$T_{C}$ obs. $^{\mathrm{a}}$ & 4.0 & 4.0 & 4.0 & 4.0 & 4.0 \\
tetracene & & & & & \\
$\mu^{*}$ & 0.069 & 0.118 & 0.163 & 0.205 & 0.244 \\
$T_{C}$ obs. $^{\mathrm{a}}$ & 2.7 & 2.7 & 2.7 & 2.7 & 2.7 \\
pentacene & & & & & \\
$\mu^{*}$ & 0.041 & 0.084 & 0.124 & 0.161 & 0.197 \\
$T_{C}$ obs. $^{\text {a }}$ & 1.9 & 1.9 & 1.9 & 1.9 & 1.9 \\
\hline \hline
\end{tabular}

${ }^{a}$ Reference 7.

the $\mathrm{C}-\mathrm{C}$ stretching $A_{g}$ modes of 1445 and $1610 \mathrm{~cm}^{-1}$ in anthracene are smaller than those for the $\mathrm{C}-\mathrm{C}$ stretching $A_{1}$ modes of 1434 and $1670 \mathrm{~cm}^{-1}$, respectively, in phenanthrene. This can be understood as follows. When anthracene is distorted along the $A_{g}$ mode of $1445 \mathrm{~cm}^{-1}$, the antibonding interactions between $\mathrm{C}_{1 \mathrm{a}}$ and $\mathrm{C}_{2 \mathrm{a}}$, and $\mathrm{C}_{1 \mathrm{~b}}$ and $\mathrm{C}_{2 \mathrm{~b}}$ in the $b_{3 u}$ LUMO are weakened, and the bonding interaction between $\mathrm{C}_{2 \mathrm{a}}$ and $\mathrm{C}_{2 \mathrm{~b}}$ is strengthened, and therefore the $b_{3 u}$ LUMO is stabilized in energy. However, the antibonding interactions between $\mathrm{C}_{3 \mathrm{a}}$ and $\mathrm{C}_{4 \mathrm{a}}$, and $\mathrm{C}_{3 \mathrm{~b}}$ and $\mathrm{C}_{4 \mathrm{~b}}$ is strengthened, and the bonding interactions between $\mathrm{C}_{2 \mathrm{a}}$ and $\mathrm{C}_{3 \mathrm{a}}, \mathrm{C}_{4 \mathrm{a}}$ and $\mathrm{C}_{4 \mathrm{~b}}$, and $\mathrm{C}_{2 \mathrm{~b}}$ and $\mathrm{C}_{3 \mathrm{~b}}$ are weakened and thus the $b_{3 u}$ LUMO is significantly destabilized in energy by such a distortion. Since such stabilization and destabilization effects are compensated each other, the $A_{g}$ mode of $1445 \mathrm{~cm}^{-1}$ couples less strongly to the LUMO in anthracene than the $A_{1}$ mode of $1434 \mathrm{~cm}^{-1}$ in phenanthrene. In addition, the frontier orbitals of anthracene is somewhat localized on $\mathrm{C}_{1 \mathrm{a}}, \mathrm{C}_{3 \mathrm{a}}$, $\mathrm{C}_{1 \mathrm{~b}}$, and $\mathrm{C}_{3 \mathrm{~b}}$, while those of phenanthrene are distributed evenly over the carbon structure. That is, the frontier orbitals of anthracene have nonbonding characters and thus these orbitals cannot strongly couple to the $A_{g}$ vibrational modes. This is also the case for other acene-edge-type hydrocarbons. The lowest frequency mode and the $\mathrm{C}-\mathrm{C}$ stretching modes strongly couple to the LUMO also in other acene- and phenanthrene-edge-type hydrocarbons.

The calculated total electron-phonon coupling constants $\left(l_{\text {LUMO }}\right)$ in the monoanions of acene- and phenanthrene- edge-type hydrocarbons are shown in Fig. 6. The coupling constants were estimated to be $0.322,0.254,0.186,0.154$, and $0.127 \mathrm{eV}$ in the monoanions of benzene, naphthalene, anthracene, tetracene, and pentacene, respectively, and 0.300, 0.194 , and $0.179 \mathrm{eV}$ in the monoanions of phenanthrene, chrysene, and benzo[a]chrysene, respectively. Therefore, the coupling constants generally decrease with an increase in the number of carbon atoms in both acene- and phenanthreneedge-type hydrocarbons. The coupling constant for the monoanion of phenanthrene $(0.300 \mathrm{eV})$ is larger than that for the monoanion of anthracene $(0.186 \mathrm{eV})$ although both molecules have the same molecular formula $\left(\mathrm{C}_{14} \mathrm{H}_{10}\right)$. Furthermore, the coupling constant for chrysene monoanion (0.194 $\mathrm{eV})$ is larger than that for tetracene monoanion $(0.154 \mathrm{eV})$, and that for benzo[a]chrysene monoanion $(0.179 \mathrm{eV})$ is larger than that for pentacene monoanion $(0.127 \mathrm{eV})$.

\section{Possible $T_{\mathrm{c}} s$ for the monoanions of phenanthrene- edge-type hydrocarbon crystals}

We are now able to estimate $T_{C}$ for the hydrocarbon monoanions by using the approximate solution of the Eliashberg equation ${ }^{32}$ from the intramolecular vibronic coupling constants, $g_{\text {LUMO }}\left(\omega_{m}\right)$, and Eqs. (7) and (8). On the basis of the calculated BCS electron-phonon coupling constants, ${ }^{33}$ we estimate $T_{\mathrm{c}} \mathrm{s}$ using McMillan's formula. ${ }^{34,35}$ McMillan's formula was derived from a three-dimensional formalism, while the electron carriers in acene-edge-type molecules

TABLE II. Calculated $T_{\mathrm{c}} \mathrm{s}(\mathrm{K})$ for the monoanion of phenanthrene $\left(\mathrm{C}_{14} \mathrm{H}_{10}\right)$ as a function of $\mu^{*}$ and $n(0)$.

\begin{tabular}{|c|c|c|c|c|c|}
\hline & $n(0)=2.0$ & $n(0)=2.5$ & $n(0)=3.0$ & $n(0)=3.5$ & $n(0)=4.0$ \\
\hline$\mu^{*}=0.00$ & 89.88 & 127.09 & 160.10 & 188.81 & 213.67 \\
\hline$\mu^{*}=0.05$ & 62.86 & 97.76 & 130.06 & 158.80 & 184.04 \\
\hline$\mu^{*}=0.10$ & 39.54 & 70.56 & 101.16 & 129.32 & 154.55 \\
\hline$\mu^{*}=0.15$ & 21.17 & 46.55 & 74.19 & 100.96 & 125.64 \\
\hline$\mu^{*}=0.20$ & 8.72 & 26.88 & 50.13 & 74.47 & 97.90 \\
\hline$\mu^{*}=0.25$ & 2.24 & 12.59 & 30.05 & 50.77 & 72.07 \\
\hline$\mu^{*}=0.30$ & 0.21 & 4.12 & 14.99 & 30.88 & 49.03 \\
\hline$\mu^{*}=0.35$ & $\ldots$ & 0.68 & 5.51 & 15.79 & 29.76 \\
\hline$\mu^{*}=0.40$ & $\ldots$ & 0.02 & 1.15 & 6.08 & 15.20 \\
\hline$\mu^{*}=0.45$ & $\ldots$ & $\ldots$ & 0.07 & 1.40 & 5.85 \\
\hline$\mu^{*}=0.50$ & $\cdots$ & $\cdots$ & $\cdots$ & 0.11 & 1.36 \\
\hline$\mu^{*}=0.55$ & $\cdots$ & $\cdots$ & $\cdots$ & $\cdots$ & 0.11 \\
\hline
\end{tabular}


TABLE III. Calculated $T_{\mathrm{c}} \mathrm{s}(\mathrm{K})$ for the monoanion of chrysene $\left(\mathrm{C}_{18} \mathrm{H}_{12}\right)$ as a function of $\mu^{*}$ and $n(0)$.

\begin{tabular}{|c|c|c|c|c|c|}
\hline & $n(0)=2.0$ & $n(0)=2.5$ & $n(0)=3.0$ & $n(0)=3.5$ & $n(0)=4.0$ \\
\hline$\mu^{*}=0.00$ & 29.27 & 50.04 & 71.55 & 92.37 & 111.88 \\
\hline$\mu^{*}=0.05$ & 14.41 & 30.55 & 49.20 & 68.38 & 87.02 \\
\hline$\mu^{*}=0.10$ & 5.08 & 15.56 & 30.17 & 46.74 & 63.78 \\
\hline$\mu^{*}=0.15$ & 0.94 & 5.86 & 15.51 & 28.45 & 43.03 \\
\hline$\mu^{*}=0.20$ & 0.04 & 1.25 & 5.96 & 14.48 & 25.70 \\
\hline$\mu^{*}=0.25$ & $\ldots$ & 0.08 & 1.33 & 5.48 & 12.72 \\
\hline$\mu^{*}=0.30$ & $\cdots$ & $\cdots$ & 0.09 & 1.20 & 4.59 \\
\hline$\mu^{*}=0.35$ & $\cdots$ & $\cdots$ & $\cdots$ & 0.08 & 0.92 \\
\hline$\mu^{*}=0.40$ & $\ldots$ & $\ldots$ & $\ldots$ & $\ldots$ & 0.05 \\
\hline
\end{tabular}

form a quasi-two-dimensional system. But we believe that McMillan's formula is still available for qualitative discussions. For $\lambda<1.5$, this is available for an approximate solution to the Eliashberg equations,

$$
T_{c}=\frac{\omega_{\ln }}{1.2} \exp \left[-\frac{1.04(1+\lambda)}{\lambda-\mu^{*}(1+0.62 \lambda)}\right],
$$

where $\mu^{*}$ is the Coulomb pseudopotential describing the electron-electron repulsion, and the logarithmically averaged phonon frequency, $\omega_{\ln },{ }^{35}$ is given by

$$
\omega_{\ln }=\exp \left\{\sum_{m} \frac{\lambda_{m} \ln \omega_{m}}{\lambda}\right\} .
$$

The density of states at the Fermi level $n(0)$ is obviously sensitive to the overlap (the transfer integral) between the LUMOs on neighboring molecules, and consequently to the distance and the orientation between the molecules and to the extent and the position of the nodes of the LUMO. Therefore, let us first estimate approximate values of $n(0)$ for anthracene, tetracene, and pentacene using Eqs. (9) and (10). $T_{\mathrm{c}} \mathrm{s}$ for the monoanions of anthracene, tetracene, and pentacene as a function of $n(0)$ and $\mu^{*}$ are listed in Table I. The $T_{c} \mathrm{~s}$ for anthracene, tetracene, and pentacene were reported to be $4,2.7$, and $1.9 \mathrm{~K}$, respectively. ${ }^{7}$ Considering usual values of $\mu^{*}(0.1-0.2)$ and the experimental values of $T_{\mathrm{c}} \mathrm{s}$, the values of $n(0)$ for anthracene, tetracene, and pentacene are 2.03.0, 2.0-3.5, and 2.5-4.0 states/eV $\cdot \mathrm{spin} \cdot \mathrm{molecule,} \mathrm{respec-}$ tively. Therefore, $n(0)$ would slightly increase with an increasing in molecular size. Calculated $T_{\mathrm{c}} \mathrm{s}$ for the monoanions of phenanthrene, chrysene, and benzo[a]chrysene as a function of $n(0)$ and $\mu^{*}$ are listed in Tables II-IV, respectively. We expect from these data that the monoanions of phenanthrene-edge-type hydrocarbons would have higher $T_{\mathrm{c}} \mathrm{s}$ than the monoanions of acene-edge-type hydrocarbons if we assume that both type hydrocarbons with the same molecular formula have approximately similar values of $n(0)$ and $\mu^{*}$. For example, the $T_{C}$ for the monoanion of phenanthrene is estimated to be high; $39.54 \mathrm{~K}$ for $n(0)=2.0$ and $\mu^{*}=0.10,46.55 \mathrm{~K}$ for $n(0)=2.5$ and $\mu^{*}=0.15$, and 50.13 $\mathrm{K}$ for $n(0)=3.0$ and $\mu^{*}=0.20$. The $T_{C}$ for the monoanion of chrysene is also estimated to be higher than that for the monoanion of tetracene; $15.56 \mathrm{~K}$ for $n(0)=2.5$ and $\mu^{*}$ $=0.10,15.51 \mathrm{~K}$ for $n(0)=3.0$ and $\mu^{*}=0.15$, and $14.48 \mathrm{~K}$ for $n(0)=3.5$ and $\mu^{*}=0.20$. Therefore, molecular edge structures as well as molecular sizes have relevance to the values of the $T_{\mathrm{c}} \mathrm{s}$.

\section{Electronic structures of anthracene and phenanthrene}

To clarify the remarkable difference in polyacene and polyphenanthrene, we examine the electronic structures of small carbon clusters with different edge structures. For example, we have investigated anthracene and phenanthrene as a member of the acene-edge group and the phenanthreneedge group, respectively. We think that the difference between the two types of ladder polymers with different edge structures can be reduced to the characterization of anthracene and phenanthrene. The characterization of the electronic structures of anthracene and phenanthrene is a classical problem in quantum chemistry. Fukui ${ }^{23}$ and Hosoya ${ }^{24,25}$ explained the reason why phenanthrene is energetically more stable than anthracene by regarding naphthalene as a starting molecule for construction of the two PAHs. To the best of our knowledge, however, detailed orbital-interaction analyses that are helpful for gaining a better understanding of the contrast between the two PAHs have not yet been performed.

Let us look at the difference between anthracene and phenanthrene in terms of orbital interactions. ${ }^{22}$ Anthracene

\begin{tabular}{|c|c|c|c|c|c|}
\hline & $n(0)=2.0$ & $n(0)=2.5$ & $n(0)=3.0$ & $n(0)=3.5$ & $n(0)=4.0$ \\
\hline$\mu^{*}=0.00$ & 20.90 & 37.36 & 55.01 & 72.53 & 89.24 \\
\hline$\mu^{*}=0.05$ & 9.29 & 21.35 & 36.10 & 51.83 & 67.50 \\
\hline$\mu^{*}=0.10$ & 2.72 & 9.76 & 20.61 & 33.66 & 47.58 \\
\hline$\mu^{*}=0.15$ & 0.34 & 3.02 & 9.43 & 18.94 & 30.32 \\
\hline$\mu^{*}=0.20$ & $\cdots$ & 0.43 & 2.94 & 8.48 & 16.59 \\
\hline$\mu^{*}=0.25$ & $\cdots$ & 0.01 & 0.43 & 2.55 & 7.11 \\
\hline$\mu^{*}=0.30$ & $\cdots$ & $\ldots$ & 0.01 & 0.35 & 1.98 \\
\hline$\mu^{*}=0.35$ & $\cdots$ & $\cdots$ & $\cdots$ & 0.01 & 0.23 \\
\hline
\end{tabular}

TABLE IV. Calculated $T_{\mathrm{c}} \mathrm{s}(\mathrm{K})$ for the monoanion of benzo[a]chrysene $\left(\mathrm{C}_{22} \mathrm{H}_{14}\right)$ as a function of $\mu^{*}$ and $n(0)$. 


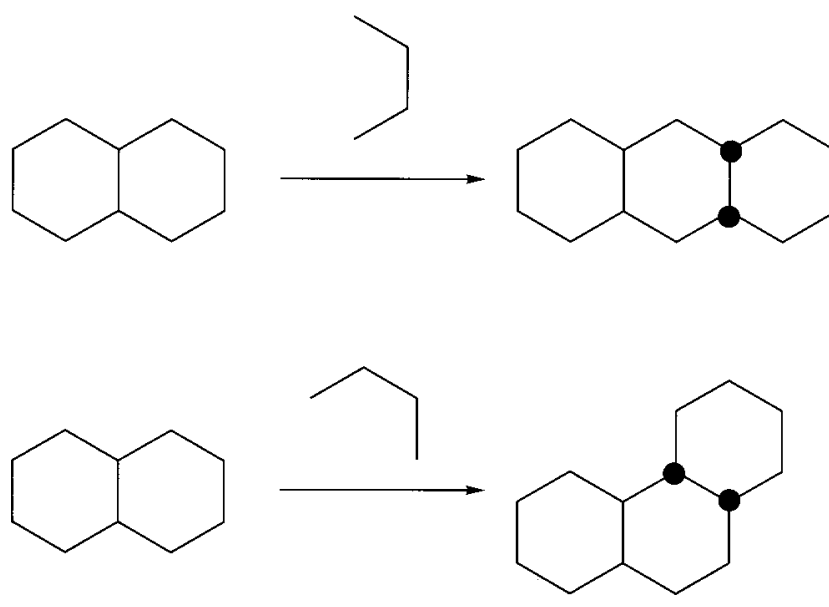

Scheme 2

and phenanthrene can be theoretically partitioned into naphthalene and butadiene fragments in different ways, although other partitions are possible. Anthracene is derived by connecting the two terminal carbons of butadiene to the 2 and 3 sites of naphthalene, and phenanthrene by connecting the

(a) anthracene

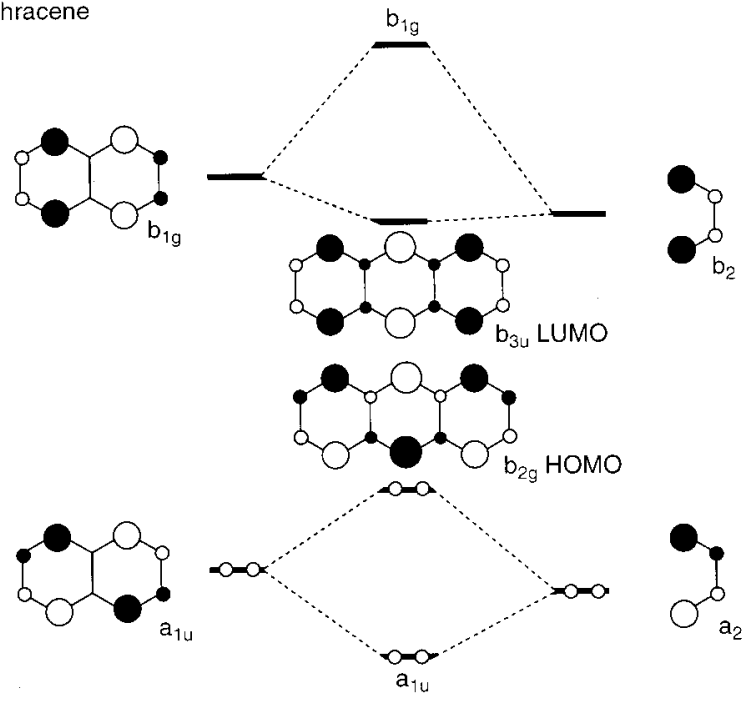

(b) phenanthrene
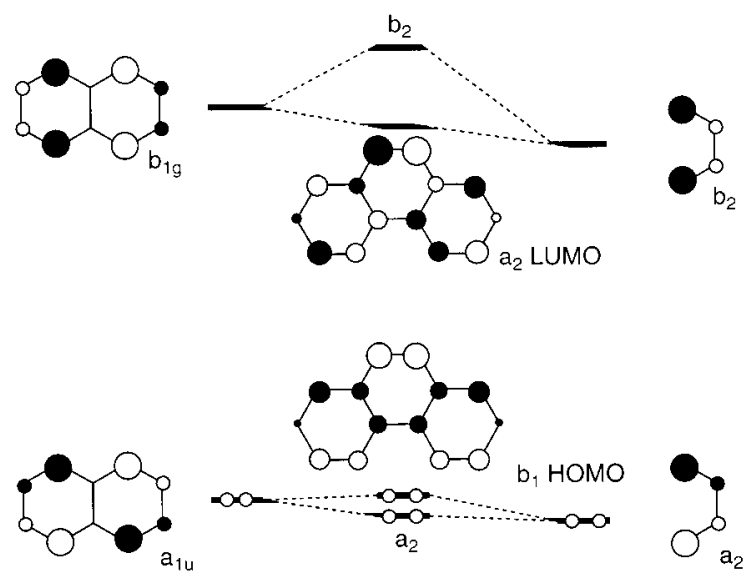

FIG. 7. Orbital-interaction diagram for (a) anthracene and (b) phenanthrene partitioned into naphthalene and butadiene fragments. two terminal carbons of butadiene to the 1 and 2 sites of naphthalene, as indicated in Scheme 2. Tetracene and chrysene can be constructed in similar ways. We apply the useful fragment molecular orbital (FMO) method ${ }^{36}$ to the characterization of the electronic structures of anthracene and phenanthrene.

We show an orbital-interaction diagram for anthracene in Fig. 7, in which $\sigma$ orbitals are neglected. It is essential to note that the $b_{2 g}$ HOMO and $b_{3 u}$ LUMO of anthracene are close-lying compared with the HOMOs and LUMOs of the naphthalene and butadiene fragments. We consider the reason why the HOMO-LUMO gap of anthracene becomes small. As shown in Fig. 7, the $a_{1 u}$ HOMO of naphthalene and the $a_{2}$ HOMO of butadiene interact nicely at the connecting sites (2 and 3 sites of naphthalene) so that the inphase combination $a_{1 u}$ is pushed down and out-of-phase counterpart $b_{2 g}$, the HOMO of anthracene, is pushed up. Moreover, since the $b_{1 g}$ LUMO of naphthalene and the $b_{2}$ LUMO of butadiene also interact nicely, the in-phase combination $b_{3 u}$, the LUMO of anthracene, is pushed down and the out-of-phase combination $b_{1 g}$ is pushed up. As a consequence, the HOMO-LUMO gap of anthracene becomes small.

On the other hand, the HOMO-LUMO gap of phenanthrene is rather large, as shown in Fig. 7. The FMO analyses for phenanthrene are strikingly different from those for anthracene. The $a_{1 u} \mathrm{HOMO}$ of naphthalene and the $a_{2} \mathrm{HOMO}$ of butadiene have no good interaction at the connecting sites ( 1 and 2 sites of naphthalene), but the $a_{1 u}$ HOMO of naphthalene and the $b_{2}$ LUMO of butadiene have a good interaction due to their orbital symmetries. The latter in-phase HOMO-LUMO interaction between the fragments would importantly give rise to electron delocalization at the connecting sites, ${ }^{23}$ which will lead to stabilization of this molecule, phenanthrene. Also, the $b_{1 g}$ LUMO of naphthalene and the $b_{2}$ LUMO of butadiene have no interaction at the connecting sites, but the $b_{1 g}$ LUMO of naphthalene and the $a_{2}$ HOMO of butadiene have a good interaction that would also lead to electron delocalization at the connecting sites. As a consequence, the HOMO of phenanthrene is stabilized and the LUMO of phenanthrene is destabilized. Thus, the HOMO-LUMO gap of phenanthrene becomes larger than that of anthracene.

\section{CONCLUDING REMARKS}

We have studied the vibronic interaction and its role in the occurrence of possible superconductivity in phenanthrene-edge-type hydrocarbons. We have computed the vibrational frequencies and the orbital vibronic coupling constants for phenanthrene-edge-type hydrocarbons. Using the vibronic coupling constants, we evaluated the electronphonon coupling constants in the monoanions of phenanthrene-edge-type hydrocarbons. Our calculational result shows that the $\mathrm{C}-\mathrm{C}$ stretching $A_{1}$ modes of 1434 and $1670 \mathrm{~cm}^{-1}$ have large electron-phonon coupling constants in the monoanion of phenanthrene. The lowest frequency $A_{g}$ mode of $399 \mathrm{~cm}^{-1}$ and the $\mathrm{C}-\mathrm{C}$ stretching modes of 1445 and $1610 \mathrm{~cm}^{-1}$ strongly couple to the $b_{3 u}$ LUMO in anthracene. As in anthracene and phenanthrene, the lowest fre- 
quency mode and the $\mathrm{C}-\mathrm{C}$ stretching modes give large electron-phonon coupling constants in the monoanions of other acene- and phenanthrene-edge-type hydrocarbons. The total electron-phonon coupling constants for the monoanions of anthracene, tetracene, and pentacene are estimated to be $0.186,0.154$, and $0.127 \mathrm{eV}$, respectively, whereas those for the monoanions of phenanthrene, chrysene, and benzo[a]chrysene are estimated to be $0.300,0.194$, and 0.179 $\mathrm{eV}$, respectively. Therefore, the electron-phonon coupling constants decrease with an increase in the number of carbon atoms in both acene- and phenanthrene-edge-type hydrocarbons. The total electron-phonon coupling constants for the monoanions of phenanthrene-edge-type hydrocarbons are larger than those for the monoanions of acene-edge-type hydrocarbons. The LUMOs of acene-edge-type hydrocarbons are localized at the edges, whereas those of phenanthreneedge-type hydrocarbons are distributed over the carbon structure. That is, the LUMOs of acene-edge-type hydrocarbons have somewhat nonbonding character and thus cannot strongly couple to the totally symmetric vibrational modes compared with the LUMOs of phenanthrene-edge-type hydrocarbons. Therefore, not only molecular sizes but also molecular edge structures have relevance to the strength of the electron-phonon coupling. We estimated possible $T_{\mathrm{c}} \mathrm{s}$ for the monoanions of phenanthrene-edge-type hydrocarbons. According to our calculation, the monoanions of phenanthreneedge-type hydrocarbons would have higher $T_{\mathrm{c}} \mathrm{s}$ than the monoanions of acene-edge-type hydrocarbons if the monoanions of phenanthrene-edge-type hydrocarbons exhibit superconductivity. Using the FMO method, we explained the distinct electronic structures of the two small PAHs with different type of edges such as anthracene and phenanthrene.

\section{ACKNOWLEDGMENTS}

K.Y. would like to thank a Grant-in-Aid for Scientific Research on the Priority Area "Molecular Physical Chemistry" from the Monbu-Kagaku-Shou and the Iwatani Naoji Foundation's Research Grant. T.K. is grateful to a Research Fellowship from the Japan Society for the Promotion of Science.

${ }^{1}$ (a) J. R. Schrieffer, Theory of Superconductivity (Addison-Wesley, Reading, MA, 1964); (b) P. G. de Gennes, Superconductivity of Metals and Alloys (Benjamin, New York, 1966).

2 (a) C. Kittel, Quantum Theory of Solids (Wiley, New York, 1963); (b) J. M. Ziman, Principles of the Theory of Solids (Cambridge University Press, Cambridge, 1972); (c) H. Ibach and H. Lüth, Solid-State Physics (Springer, Berlin, 1995).

${ }^{3}$ G. Grimvall, The Electron-Phonon Interaction in Metals (North-Holland, Amsterdam, 1981)

${ }^{4} \mathrm{G}$. Fischer, Vibronic Coupling: The Interaction between the Electronic and Nuclear Motions (Academic, London, 1984).

${ }^{5}$ (a) I. B. Bersuker, The Jahn-Teller Effect and Vibronic Interactions in Modern Chemistry (Plenum, New York, 1984); (b) I. B. Bersuker and V. Z. Polinger, Vibronic Interactions in Molecules and Crystals (Springer, Berlin, 1989).
${ }^{6}$ J. H. Schön, Ch. Kloc, R. C. Haddon, and B. Batlogg, Science 288, 656 (2000).

${ }^{7}$ J. H. Schön, Ch. Kloc, and B. Batlogg, Nature (London) 406, 702 (2000).

${ }^{8}$ A. Dodabalapur, L. Torsi, and H. E. Katz, Science 268, 270 (1995).

${ }^{9}$ J. H. Schön, Ch. Kloc, and B. Batlogg, Science 287, 1022 (2000).

${ }^{10}$ E. A. Silinch and V. Capek, Organic Molecular Crystals (AIP, New York, 1994).

${ }^{11}$ W. Warta and N. Karl, Phys. Rev. B 32, 1172 (1985).

${ }^{12}$ W. Warta, R. Stehle, and N. Karl, Appl. Phys. A: Solids Surf. A36, 163 (1985).

${ }^{13}$ S. Kivelson and O. L. Chapman, Phys. Rev. B 28, 7236 (1983).

${ }^{14}$ A. Mishima and M. Kimura, Synth. Met. 11, 75 (1985).

${ }^{15}$ J. H. Schön, Ch. Kloc, and B. Batlogg, Nature (London) 408, 549 (2000).

${ }^{16}$ (a) C. M. Varma, J. Zaanen, and K. Raghavachari, Science 254, 989 (1991); (b) M. Lannoo, G. A. Baraff, M. Schlüter, and D. Tomanek, Phys. Rev. B 44, 12106 (1991); (c) M. Schluter, M. Lannoo, M. Needels, G. A. Baraff, and D. Tomanek, Phys. Rev. Lett. 68, 526 (1992); (d) Y. Asai and Y. Kawaguchi, Phys. Rev. B 46, 1265 (1992); (e) J. C. R. Faulhaber, D. Y. K. Ko, and P. R. Briddon, ibid. 48, 661 (1993); (f) V. P. Antropov, O. Gunnarsson, and A. I. Liechtenstein, ibid. 48, 7651 (1993); (g) A. Auerbach, N. Manini, and E. Tosatti, ibid. 49, 12998 (1994); (h) N. Manini, E. Tosatti, and A. Auerbach, ibid. 49, 13008 (1994); (i) O. Gunnarsson, ibid. 51, 3493 (1995); (j) K. Tanaka, Y. Huang, and T. Yamabe, ibid. 51, 12715 (1995); (k) J. L. Dunn and C. A. Bates, ibid. 52, 5996 (1995); (1) O. Gunnarsson, Rev. Mod. Phys. 69, 575 (1997); (m) A. Devos and M. Lannoo, Phys. Rev. B 58, 8236 (1998); (n) O. Gunnarsson, Nature (London) 408, 528 (2000).

${ }^{17}$ (a) B. A. Hess, Jr. and L. J. Schaad, J. Am. Chem. Soc. 93, 305 (1971); (b) J. Org. Chem. 36, 3418 (1971).

${ }^{18}$ (a) W. C. Herdon, Tetrahedron 29, 3 (1973); (b) W. C. Herdon and M. L. Ellzey, J. Am. Chem. Soc. 96, 6631 (1974); (c) W. C. Herdon, J. Org. Chem. 40, 3583 (1975); (d) 46, 2119 (1981); (e) Tetrahedron 38, 1389 (1982).

19 (a) S. E. Stein and R. L. Brown, Carbon 23, 105 (1985); (b) J. Am. Chem. Soc. 109, 3721 (1987); (c) R. H. Chen, S. A. Kafafi, and S. E. Stein, ibid. 111, 1418 (1989); (d) S. E. Stein and R. L. Brown, ibid. 113, 787 (1991); (e) S. E. Stein, Acc. Chem. Res. 24, 350 (1991).

${ }^{20}$ A. Klimkans and S. Larsson, Chem. Phys. 189, 25 (1994).

${ }^{21}$ (a) T. Yamabe, S. Yamashita, H. Yamabe, K. Fukui, and K. Tanaka, Collect. Czech. Chem. Commun. 53, 1881 (1988); (b) K. Yoshizawa, K. Okahara, T. Sato, K. Tanaka, and T. Yamabe, Carbon 32, 1517 (1994).

${ }^{22}$ K. Yoshizawa, K. Yahara, K. Tanaka, and T. Yamabe, J. Phys. Chem. B 102, 498 (1998).

${ }^{23}$ K. Fukui, Science 218, 747 (1982).

${ }^{24} \mathrm{H}$. Hosoya and T. Yamaguchi, Tetrahedron Lett. 52, 4659 (1975).

${ }^{25} \mathrm{H}$. Hosoya, Chemistry of Novel Aromatic Systems (Chemical Society of Japan, Tokyo, 1977) (in Japanese).

${ }^{26}$ (a) T. Kato and T. Yamabe, in Proceeding of the Fifth Symposium on Atomic-scale Surface and Interface Dynamics, 2001, p. 467; (b) T. Kato, M. Kondo, K. Yoshizawa, and T. Yamabe, Synth. Met. (in press).

${ }^{27}$ E. M. Conwell, Phys. Rev. B 22, 1761 (1980).

${ }^{28}$ (a) A. D. Becke, Phys. Rev. A 38, 3098 (1988); (b) J. Chem. Phys. 98, 5648 (1993)

${ }^{29}$ C. Lee, W. Yang, and R. G. Parr, Phys. Rev. B 37, 785 (1988).

${ }^{30}$ (a) R. Ditchfield, W. J. Hehre, and J. A. Pople, J. Chem. Phys. 54, 724 (1971); (b) P. C. Hariharan and J. A. Pople, Theor. Chim. Acta 28, 213 (1973).

${ }^{31}$ M. J. Frisch, G. W. Trucks, H. B. Schlegel et al., GAUSSIAN 98, Gaussian, Inc., Pittsburgh, PA, 1998.

${ }^{32}$ (a) G. M. Eliashberg, Zh. Eksp. Teor. Fiz. 38, 966 (1960); (b) 39, 1437 (1960); (c) Sov. Phys. JETP 11, 696 (1960); (d) 12, 1000 (1961).

${ }^{33}$ P. B. Allen, Phys. Rev. B 6, 2577 (1972).

${ }^{34}$ W. L. McMillan, Phys. Rev. 167, 331 (1968).

${ }^{35}$ M. Schlüter, M. Lannoo, M. Needels, G. A. Baraff, and D. Tomanek, J. Phys. Chem. Solids 53, 1473 (1992).

${ }^{36}$ T. A. Albright, J. K. Burdett, and M. -H. Whangbo, Orbital Interactions in Chemistry (Wiley, New York, 1985). 